\title{
Ginseng and Anticancer Drug Combination to Improve Cancer Chemotherapy: A Critical Review
}

\author{
Shihong Chen,,2 Zhijun Wang, ${ }^{2}$ Ying Huang, ${ }^{3}$ Stephen A. O'Barr, ${ }^{3}$ Rebecca A. Wong, \\ Steven Yeung, ${ }^{2}$ and Moses Sing Sum Chow ${ }^{2}$ \\ ${ }^{1}$ Department of Pharmacy, Xiamen Medical College, Xiamen, Fujian 361008, China \\ ${ }^{2}$ Center for Advancement of Drug Research and Evaluation, College of Pharmacy, Western University of Health Sciences, \\ Pomona, CA 91766, USA \\ ${ }^{3}$ Department of Pharmaceutical Sciences, College of Pharmacy, Western University of Health Sciences, Pomona, CA 91766, USA
}

Correspondence should be addressed to Moses Sing Sum Chow; msschow@westernu.edu

Received 16 December 2013; Revised 17 March 2014; Accepted 20 March 2014; Published 30 April 2014

Academic Editor: Zhong Zuo

Copyright (C) 2014 Shihong Chen et al. This is an open access article distributed under the Creative Commons Attribution License, which permits unrestricted use, distribution, and reproduction in any medium, provided the original work is properly cited.

\begin{abstract}
Ginseng, a well-known herb, is often used in combination with anticancer drugs to enhance chemotherapy. Its wide usage as well as many documentations are often cited to support its clinical benefit of such combination therapy. However the literature based on objective evidence to make such recommendation is still lacking. The present review critically evaluated relevant studies reported in English and Chinese literature on such combination. Based on our review, we found good evidence from in vitro and in vivo animal studies showing enhanced antitumor effect when ginseng is used in combination with some anticancer drugs. However, there is insufficient clinical evidence of such benefit as very few clinical studies are available. Future research should focus on clinically relevant studies of such combination to validate the utility of ginseng in cancer.
\end{abstract}

\section{Introduction}

The concept of herb-herb or herb-drug combination to enhance therapeutic benefit has been utilized and practiced in China according to Chinese medicine principles for more than 2000 years [1]. Recently, herbs in combinations with anticancer drugs have been found to be capable of resensitizing the chemoresistance developed from repeated use of the anticancer drug [2]. Thus, the use of herb-drug combination to enhance therapeutic effect is of great interest, especially in cancer chemotherapy.

Among many herbs touted to improve cancer treatment, none has probably enjoyed as much worldwide reputation and interest as ginseng. Ginseng is widely used and is included in the pharmacopoeias in China, Japan, Germany, France, Austria, and the United Kingdom. In Asian countries and Western Europe, it is widely available as an over-thecounter drug and also commonly used as an adjuvant for cancer therapy $[3,4]$. In the US, ginseng is one of the most frequently purchased herbs; it is available and used as a dietary or an herbal supplement but not as a drug approved by the Food and Drug Administration [5]. It is consumed regularly by more than 6 million Americans [6], as the second top-selling herbal supplement (US \$62 million in annual sales in 2000 and about US $\$ 83$ million in 2010) [7, 8]. In 2002, a national survey of men and women in the US has estimated that $4-5 \%$ of those aged 45-64 years had used ginseng [9].

Although ginseng consumption is not limited for in cancer patients, its benefit in cancer appears to be well accepted. Common reasons for the use of ginseng by cancer patients are to improve clinical outcomes, enhance quality of life, treat cancer-related symptoms, reduce adverse effects of chemotherapy, and potentially enhance the effects of chemotherapeutic agents. In addition, ginseng may exert a chemopreventive action: an epidemiological study has shown that patients taking ginseng had a 50\% lower risk of cancer recurrence compared to patients not taking ginseng [10].

In view of its wide usage and potential benefit when used in combination with anticancer drugs, the present paper intends to critically review the evidence of such 
benefit as well as potential mechanisms involved. Although numerous reviews on ginseng-drug interaction have been already published $[47,48]$, no article has critically reviewed ginseng-anticancer drug combination for improvement of chemotherapy.

Both English and Chinese publications on ginseng and anticancer drug combination to improve cancer chemotherapy were searched from the Medline database (1990 2013) and China Academic Journals Database (1983 2013), respectively. All articles from in vitro, in vivo animal models, and human studies on the combination of ginseng or its active components with chemotherapeutic agent for anticancer effect were included. Because there are several types of ginseng with different active components, their general properties are briefly discussed to provide relevant background information before reviewing the specific combination in order to provide better understanding of the rationale of such combination.

\section{Different Types of Ginseng and Its Preparation}

Ginseng is a perennial herb that belongs to the Araliaceae family and Panax genus [49]. The root is the preferred part of the plant due to the presence of active components (see below), and the species most commonly used are Panax ginseng C.A. Meyer (Asian ginseng or Korean ginseng) and Panax quinquefolius (American ginseng). Panax ginseng C.A. Meyer is usually cultivated in China and Korea and has been used as a medicinal herb in China, Japan, and Korea for thousands of years. Its commonly claimed health benefits include immunity enhancement, stress relief, and prevention of aging. Panax quinquefolius, originally grown in United States and Canada, has been used by Native Americans for hundreds of years. So far, majority of research on ginseng has been on Panax ginseng C.A. Meyer [5, 17].

Panax ginseng C.A. Meyer is usually harvested after 4 to 6 years of cultivation and is classified into three types based on processing methods: (1) fresh (less than 4 years old, consumed in its fresh state), (2) white ginseng (4 to 6 years old, typically air or oven dried after peeling), (3) red ginseng ( 6 years old, steamed prior to drying, without peeling). These processing methods are intended to improve efficacy, safety, and preservation [50]. Recently, a new heat-processed ginseng, called Sun ginseng (SG), has been prepared by steaming with white ginseng at high temperature and pressure. Sun ginseng has been reported to contain more unique ginsenosides than the red ginseng. A preparation containing Sun ginseng extract with specific standardization is now available as functional food in Korea [21, 22, 24].

Many ginseng products are available on the market as fresh slices, juice, extract (tincture or boiled), powder, tea, tablet, capsule, and other forms. Two-year-old fresh ginseng is also used as an ingredient in Korean chicken-ginseng soup (boiled chicken with young ginseng root), samketang [51]. The traditional Chinese ginseng preparation widely used clinically in China is Shengmai which consists of red ginseng, lilyturf root, and magnolia vine fruit $[28,40]$.
The quality of ginseng is believed to vary with the age at harvest. When ginseng is harvested at the time of 5 to 6 years, it is considered the "best" with ginsenoside content at its highest [52]. According to several laboratory investigations, the quality of commercially available ginseng products can vary considerably. Negative trial results may be due to poor product quality rather than lack of efficacy [35]. Thus, evaluation of study results must take product quality control into consideration.

\section{Active Components of Ginseng Relevant to Anticancer Effect}

Ginseng contains various active components including ginsenosides, polysaccharides, flavonoids, volatile oils, amino acid, and vitamins. Of these active components, ginsenosides and ginseng polysaccharides appear to be responsible for the anticancer effect [8].

Ginsenosides are the main pharmacologically active ingredients responsible for the four major actions of ginseng: vasorelaxation, antioxidation, anti-inflammation, and anticancer effect. Ginsenosides, being amphipathic in nature, are steroidal saponins that contain four transring rigid steroid skeleton. They differ from each other mainly by the number, type, and location of their sugar moieties. Thus far, more than 40 different ginsenosides have been identified and isolated. Ginsenosides can be classified into three groups based on the chemical structure of aglycones: (1) protopanaxadiol group (PPD) or diols, for example, Rb1, Rb2, Rb3, Rc, Rd, Rg3, and Rh2; (2) protopanaxatriol group (PPT) or triols, for example, Re, Rf, Rg1, Rg2, and Rh1; (3) oleanane group: only Ro $(0.6 \%$ of all ginsenosides $)[53,54]$. The total percentage of ginsenosides (w/w) can vary from $1.9 \%$ to $8.1 \%$ in ginseng root preparations [17]. Red ginseng can possess higher activity than white ginseng, due to the presence of unique ginsenosides (Rg3, Rg5, Rg6, Rh2, Rh3, Rh4, Rs3, and F4) produced during steaming method $[15,18,24]$. The relative amounts of ginsenosides may also be used to differentiate Panax species. For example, American ginseng has little or no Rf, and Panax ginseng has higher levels of Rg1 but lower levels of Rb1 (or higher ratio of Rg1/Rb1) compared to those of American ginseng [5, 49, 55, 56]. Ginsenosides are also used as marker compounds for ginseng quality control, of which Rg1, Rc, Rd, Re, Rb1, and Rb2 are quantitatively the most important and prevalent. According to a Ginseng Evaluation Program led by the American Botanical Council of Austin, Texas, Rb1, Rb2, Rc, Rd, Re, and Rg1 account for $>90 \%$ of the total ginsenoside content of the Panax ginseng root, whereas, $\mathrm{Rb} 1, \mathrm{Rb} 3, \mathrm{Rc}, \mathrm{Rd}, \mathrm{Re}$, and Rg1 make up more than $70 \%$ of total ginsenoside content in American ginseng [8]. Each ginsenoside may differ in pharmacology and mechanisms due to its different chemical structure.

Researchers are now focusing on using purified individual ginsenosides to reveal the specific mechanism of action instead of using whole ginseng root extracts. The most commonly studied ginsenosides are Rb1, Re, Rg1, Rg3, and Rh1 [5]. The relevant ginsenosides (Rb1, Rg1, Rg3, and 
Rh1) for anticancer activity and corresponding molecular mechanisms are listed in Table 1.

Besides ginsenosides, ginseng polysaccharides also possess antitumor effect through modulation of innate immunity. Ginseng polysaccharides (present in $15 \%$ of ginseng root), including neutral and acidic polysaccharides, are water-soluble. It has been reported that Panax ginseng polysaccharides contain starch-like polysaccharide and pectin and can be fractionated into two neutral (WGPN and WGPA-N) and six acidic fractions (WGPA-1-RG, WGPA-2RG, WGPA-1-HG, WGPA-2-HG, WGPA-3-HG, and WGPA$4-\mathrm{HG}$ ) by a combination of ethanol precipitation, ion exchange, and gel permeation chromatographies [57]. Many immunological studies have been performed with crude polysaccharide fractions, which are usually prepared by ethanol precipitation after extracting ginseng root with hot water. These polysaccharides have been reported to exert antitumor activity by regulating the immune response of the host organism. Using lymphocyte proliferation assays, both polysaccharides have been found to be potent $\mathrm{B}$ and $\mathrm{T}$ cell stimulators [57]. The acidic polysaccharides $(10,000-$ $150,000 \mathrm{MW}$ ), being readily soluble in water, are thought to be more active than neutral ones [27, 31,32]. Ginseng pectin has also been shown to inhibit the actions of galectin3 , a $\beta$-galactoside-binding protein associated with cancer progression [58]. Nonsaponin constituents (immunomodulating polysaccharides) and the harmonizing constituents still remain to be explored.

\section{Effects of Ginseng in Combination with Anticancer Drugs}

4.1. In Vitro Studies. A number of in vitro studies have shown an enhanced anticancer effect when the ginseng extract or its active component is combined with a chemotherapeutic agent (see Table 2). One specific effect is increasing the cytotoxicity of chemotherapeutic agents.

Ginseng extracts, including ginsenosides, have been found to enhance the cytotoxicity of several chemotherapeutic agents such as 5-fluorouracil (5-FU, an antimetabolite), irinotecan (a plant alkaloid), mitomycin C (an antibiotics), docetaxel (a taxane agent belonging to a plant alkaloid), cisplatin (an alkylating agent), and others at the concentration range of $0.1-300 \mu \mathrm{g} / \mathrm{mL}$ (see Table 2). One ginsenoside, $\mathrm{Rg} 3$, has been found to inhibit growth of various human tumor cells, such as prostate cancer cells (LNCaP, PC-3, and DU145), Lewis lung cancer cells, colon cancer cells (SW620 and HCT116), and B16 melanoma cells. Ginseng has been also found to enhance the cytotoxicity of docetaxel, cisplatin, and doxorubicin at low doses [21, 22]. Inhibition of nuclear factor-kappa (NF- $\boldsymbol{k}$ B) may be one of the potential mechanisms of the observed effect. NF- $\kappa$ B mediates tumor promotion, angiogenesis, metastasis, and resistance to chemotherapeutics through the expression of genes participating in malignant transformation and tumor promotion. Kim and coworkers have found that Rg3 can suppress the expression of several antiapoptosis genes (Bcl2, Cox-2, c-Fos, c-Jun, cyclin D1, etc.) via inhibiting NF- $\boldsymbol{\kappa} B$ and thus enhancing the susceptibility of colon cancer cells to docetaxel and other chemotherapeutics [21, 22].

Also, panaxadiol, a pseudoaglycone of diol-type ginsenoside, has been found to enhance the anticancer effects of some anticancer drugs through the regulation of cell cycle transition and the induction of apoptotic cells. Apoptosis, highly regulatory process of programmed death involving the caspase protease family, is considered to be a key factor. Apoptosis may play an important role in the panaxadiol enhanced antiproliferative effects of irinotecan on human colorectal cancer cells as well as when used in combination with 5-FU $[18,20]$.

Furthermore, the synergistic inhibitory effect of Panax ginseng when combined with $5-\mathrm{FU}$ has been observed in human gastric cancer cell line BGC823 [16]. This appears to involve $\mathrm{NO}$ which has been found to directly suppress the growth of BGC823 cells by inducing G0/G1 phase arrest through the regulation of Akt signaling pathway. Ginsenosides may increase NO production by inducing endothelial nitric oxide synthase (eNOS) phosphorylation via the ERmediated PI3-kinase/Akt pathway [16].

One major difficulty in cancer chemotherapy is the development of broad anticancer drug resistance by tumor cells. This phenomenon has been termed multidrug resistance (MDR) [8]. The overexpression of P-glycoprotein (Pgp) or the multidrug resistance-associated proteins (MRPs) confer MDR to cancer cells. Ginseng extracts may induce chemosensitization of conventional anticancer agents via downregulation of MDR-1 expression (Pgp inhibition) [26, 28]. Choi et al. found that protopanaxatriol ginsenosides can potentially reverse Pgp-mediated MDR by increasing the intracellular accumulation of drugs through competitive inhibition of Pgp [26]. In addition, Shengmai can enhance the sensitivity of cancer cells (human lung carcinoma A549, gastric carcinoma SGC-7901, breast carcinoma MCF-7, and hepatocellular carcinoma HepG-2) to various anticancer drug such as gemcitabine (an antimetabolite), cisplatin, paclitaxel (a taxane agent belonging to a plant alkaloid), and epirubicin (an antibiotics) via downregulation of the mRNA level of MDR-1 [28].

Another benefit of ginseng when used with the anticancer drug is a potential reduction in drug induced toxicity. Baek et al. have found ginsenosides $\mathrm{Rh} 4$ and $\mathrm{Rk} 3$, the active principles of Sun ginseng (SG), to significantly reduce the cisplatininduced nephrotoxicity in LLC-PK1 cells in a dose-dependent manner. The mechanisms of function and structure-activity relationships with other ginsenosides remain to be investigated [24]. Ginsenoside Rd may also ameliorate cisplatininduced renal injury, a process in which apoptosis may play a central role [25].

A critical concern in the above in vitro studies is the relatively high concentration of ginseng extract/active components used (up to $300 \mu \mathrm{g} / \mathrm{mL}$ ). Such a high concentration is likely not achievable in vivo, as the $C_{\max }$ of some ginsenosides following oral administration in rats has shown to be less than $0.7 \mu \mathrm{g} / \mathrm{mL}[59,60]$. Verification of in vitro benefit from lower concentrations of ginseng or from in vivo studies will be essential. 
TABle 1: Anticancer activities of commonly studied relevant ginsenosides [5, 8, 11-14].

\begin{tabular}{|c|c|c|}
\hline Ginsenoside & Anticancer activity & Molecular mechanism \\
\hline $\mathrm{Rb} 1$ & $\begin{array}{l}\text { Weakly antiproliferative; } \\
\text { antiangiogenic }\end{array}$ & $\begin{array}{l}\text { (i) Inhibit capillary genesis } \\
\text { (ii) Inhibit TNF-a release } \\
\text { (iii) Protect against oxidative stress } \\
\text { (iv) Inhibit tube-like structure formation of endothelial cells by regulating pigment } \\
\text { epithelium-derived factor (PEDF) through estrogen receptor- } \beta[13,14]\end{array}$ \\
\hline $\mathrm{Rb} 3$ & No antiproliferative activity & Inhibit TNF- $\alpha$ release \\
\hline $\operatorname{Rg} 1$ & Antiproliferative & $\begin{array}{l}\text { (i) Inhibit oncogenes c-myc, c-fos } \\
\text { (ii) Downregulate nucleophosmin. }\end{array}$ \\
\hline $\operatorname{Rg} 3$ & $\begin{array}{l}\text { Antiproliferative, apoptotic, } \\
\text { antiangiogenic, } \\
\text { antimetastatic, anti-invasive, } \\
\text { and cell cycle regulation, } \\
{[8,11,12]}\end{array}$ & $\begin{array}{l}\text { (i) Regulate mitochondrial cytochrome C, poly ADP ribose polymerase (PARP) and C9 } \\
\text { (ii) Inhibit MMP-2 and } 9 \\
\text { (iii) Inhibit adhesion of metastatic cells to basement membrane } \\
\text { (iv) Inhibit MDR (most potent among all ginsenosides) }\end{array}$ \\
\hline Rhl & $\begin{array}{l}\text { Causes differentiation of } \\
\text { teratocarcinoma cells, } \\
\text { strongly apoptotic }\end{array}$ & $\begin{array}{l}\text { (i) Bind to steroid receptor } \\
\text { (ii) Inhibit TNF- } \alpha \\
\text { (iii) Inhibit phosphorylation of JAK1, STAT1, STAT3, and ERK }\end{array}$ \\
\hline
\end{tabular}

4.2. In Vivo Animal Studies. A number of positive benefits have been delineated with ginseng and anticancer drug combinations. They include increase of drug exposure, inhibition of the angiogenesis and metastasis, survival benefit, reduction of side effects of anticancer drugs, and therapeutic improvement (see Table 3).

A pharmacokinetic interaction leading to enhancement of certain anticancer drugs has been reported. After pretreatment with $3.0 \mathrm{mg} / \mathrm{kg}$ Panax ginseng extract orally twice daily for ten consecutive days, the elimination half-life of 5-FU has been shown to significantly increase by approximately $58.8 \%$ (79.17 versus 125.72). The increase in $t_{1 / 2}$ caused by Panax ginseng extract can result in a higher drug exposure of 5-FU, which may lead to a longer drug effect [16]. The specific mechanism however is not known. Ginseng may increase the exposure of other drugs including docetaxel. In vitro studies using human liver microsomes have suggested that ginseng as well as its various ginsenosides, at clinically relevant concentrations, can moderately inhibit CYP1A1, CYP1A2, CYP1B1, CYP2D6, CYP2C9, CYP2C19, CYP2E1, and CYP3A4 [61].

Angiogenesis, the process of pathological vascular ingrowth critical for tumor expansion, is now known to play an important role in both growth and metastasis of some cancers. Ginsenoside Rg3 has been found to inhibit tumor angiogenesis. Combined therapy with $\mathrm{Rg} 3$ and lowdose gemcitabine or cyclophosphamide has been found to produce significant antiangiogenic effect without overt toxicity. The combined therapy has been shown to decrease vascular endothelial growth factor (VEGF) expression and microvascular density as well as blood flow in tumors (by color Doppler flow imaging) and peak systolic velocity when compared with the control mice. The combined therapy may have selectively enhanced the damage or cytotoxic effects of chemotherapy on newly formed blood vessels while simultaneously reduced Ki-67, VEGF, bcl-2, and P53 gene expression which may partially be responsible for their antiangiogenic and antitumor effects [11, 34, 36].
Survival benefit has been reported with the combination of ginseng or its active components with anticancer agents. In one study, combination treatment with paclitaxel (5 or $15 \mathrm{mg} / \mathrm{kg}$ ) and acidic polysaccharide $(25 \mathrm{mg} / \mathrm{kg})$ has resulted in a 28.6 or $42.8 \%$ increase in 30 -day life span of ICR mice bearing sarcoma 180 tumor cells, compared to paclitaxel treatment alone [27]. In another study, up to $53 \%$ of the $\mathrm{BALB} / \mathrm{c}$ mice treated with combination of cyclophosphamide and an acidic polysaccharide $(25 \mathrm{mg} / \mathrm{kg})$ have shown an increase in survival rate compared with only $10 \%$ with cyclophosphamide alone [32]. At least 3 positive survival studies have been reported with ginsenoside $\mathrm{Rg} 3$ alone or in combination with anticancer drug: (1): treatment with $\mathrm{Rg} 3$, cyclophosphamide, or their combination in athymic mice bearing human ovarian cancer SKOV-3 has been found to improve survival 23.72, 25.90, and 27.12 days, respectively, compared to 13.6 days with the control [34]. (2): combination with gemcitabine has been found to increase survival rate (100\%) compared with control or gemcitabine $(60 \%$ or $70 \%)$ in 18 days after treatments [11]. (3): in 50\% mice that survived cancer cell implantation, cyclophosphamide (low-dose), Rg3 alone, and their combination treatment groups result in 70,77 , and 95 days compared to only 29 days survival in the control group. The Rg3 anticaner drug combination treatment has shown to induce the longest survival [36]. The mechanism of such benefit however is not clear.

The combination of ginseng and various anticancer drugs have been found to lessen the reduction of weight loss, nausea/vomiting, diaphragm muscle toxicity, immunosuppression, and liver and renal function deterioration (see Table 3). Ginseng extract may decrease the side effect of weight loss from anticancer drugs by increasing the protein and RNA contents of muscles and liver in rats $[11,16,31$, $36,62]$. In addition, Ge and coworkers have found the effect of Shengmai (Chinese herbal preparation consisting of red ginseng, lilyturf root, and magnolia vine fruit) to be capable of protecting diaphragm muscles from doxorubicin induced toxicity which appears to be correlated with a decrease in 


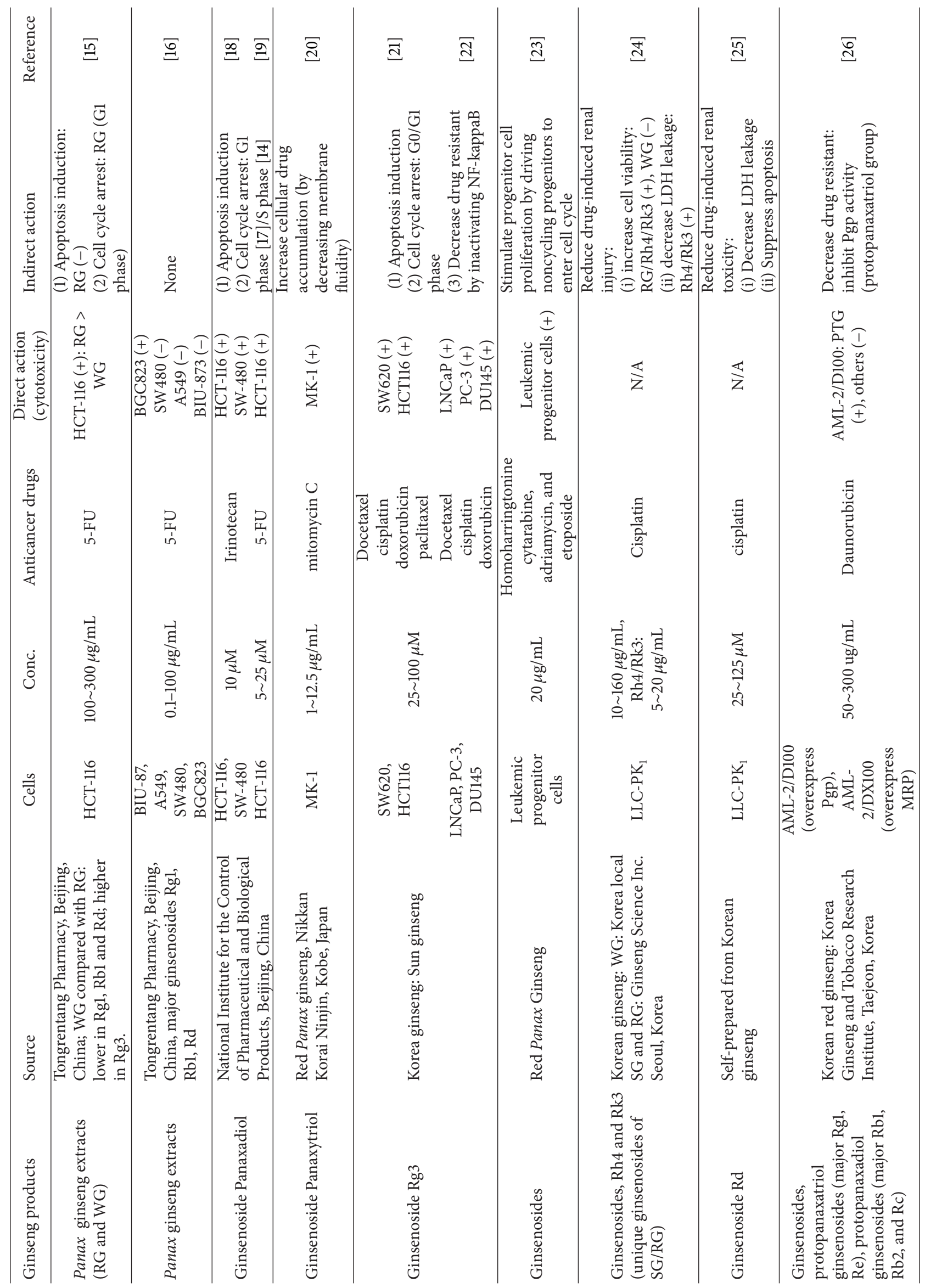




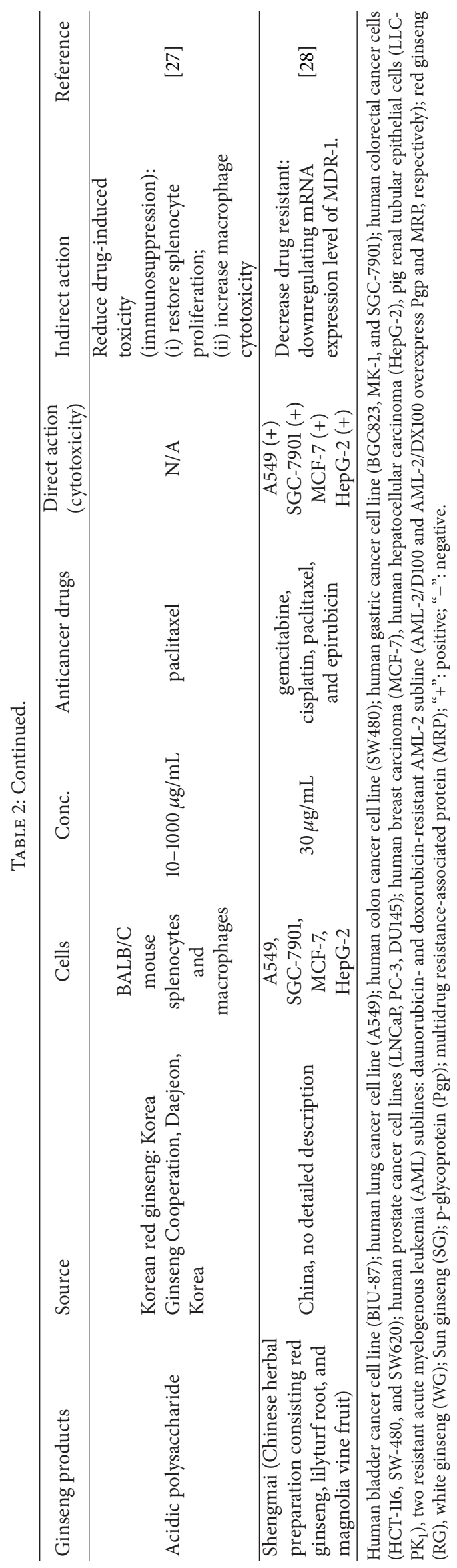




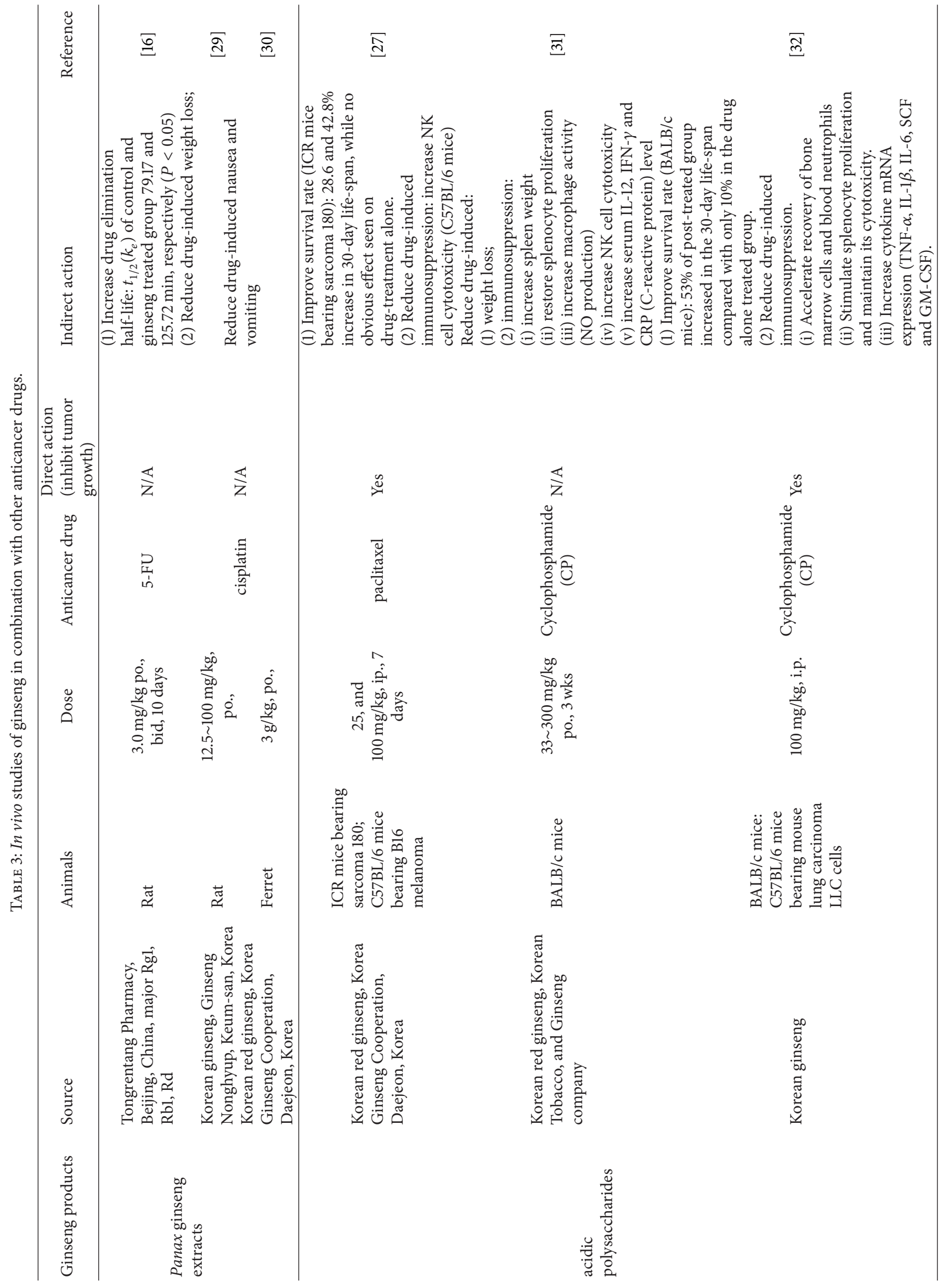




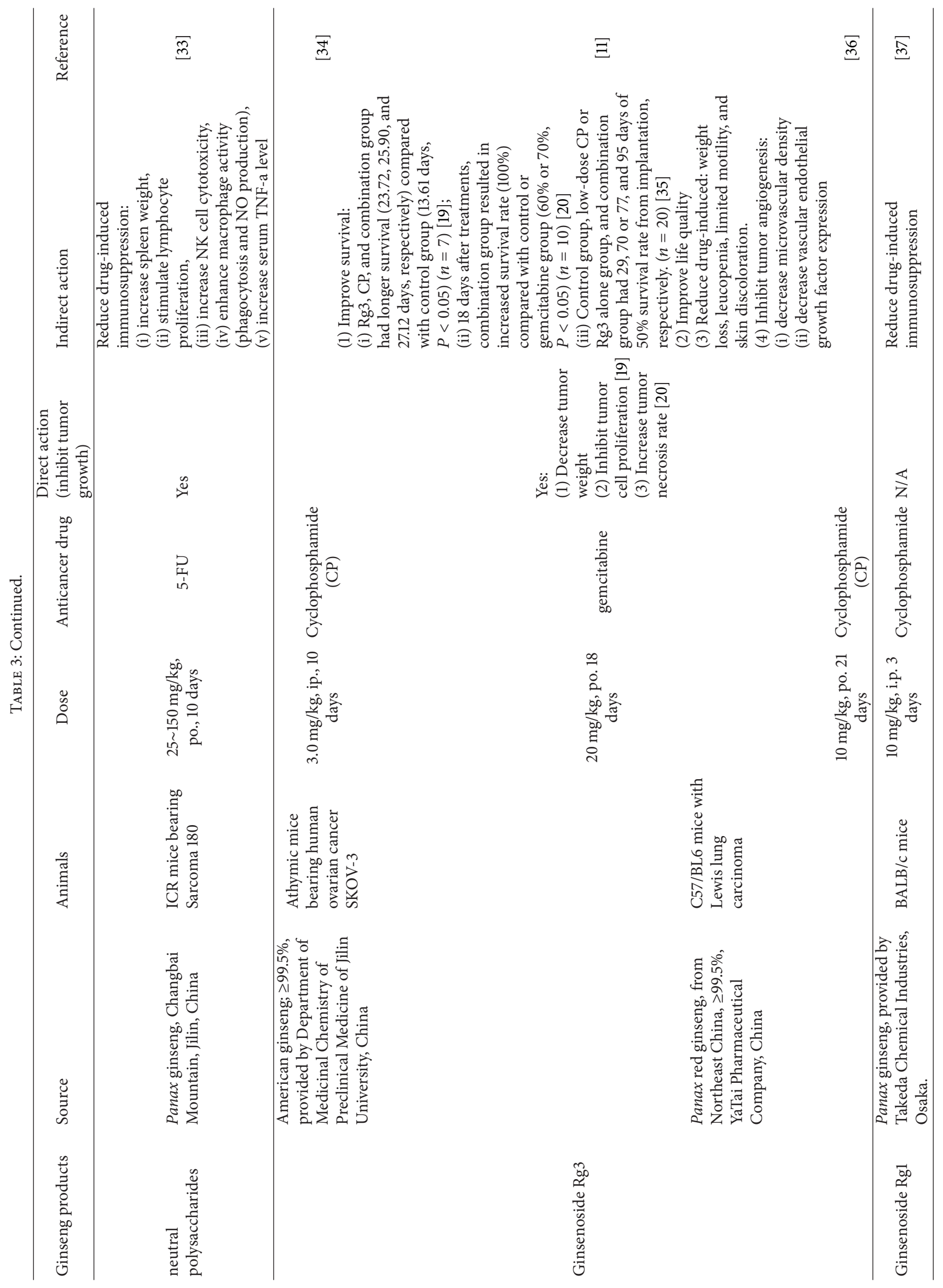




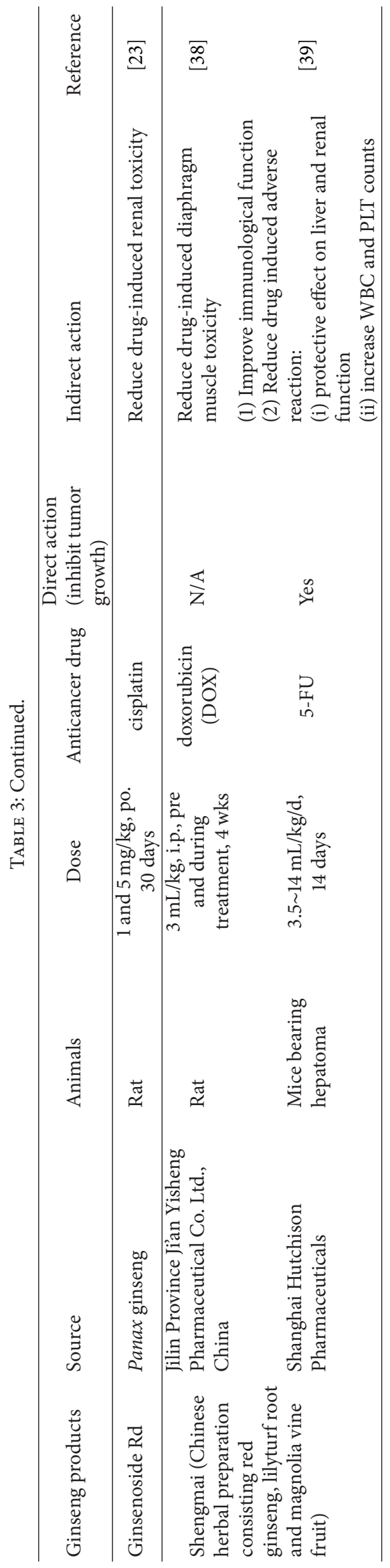




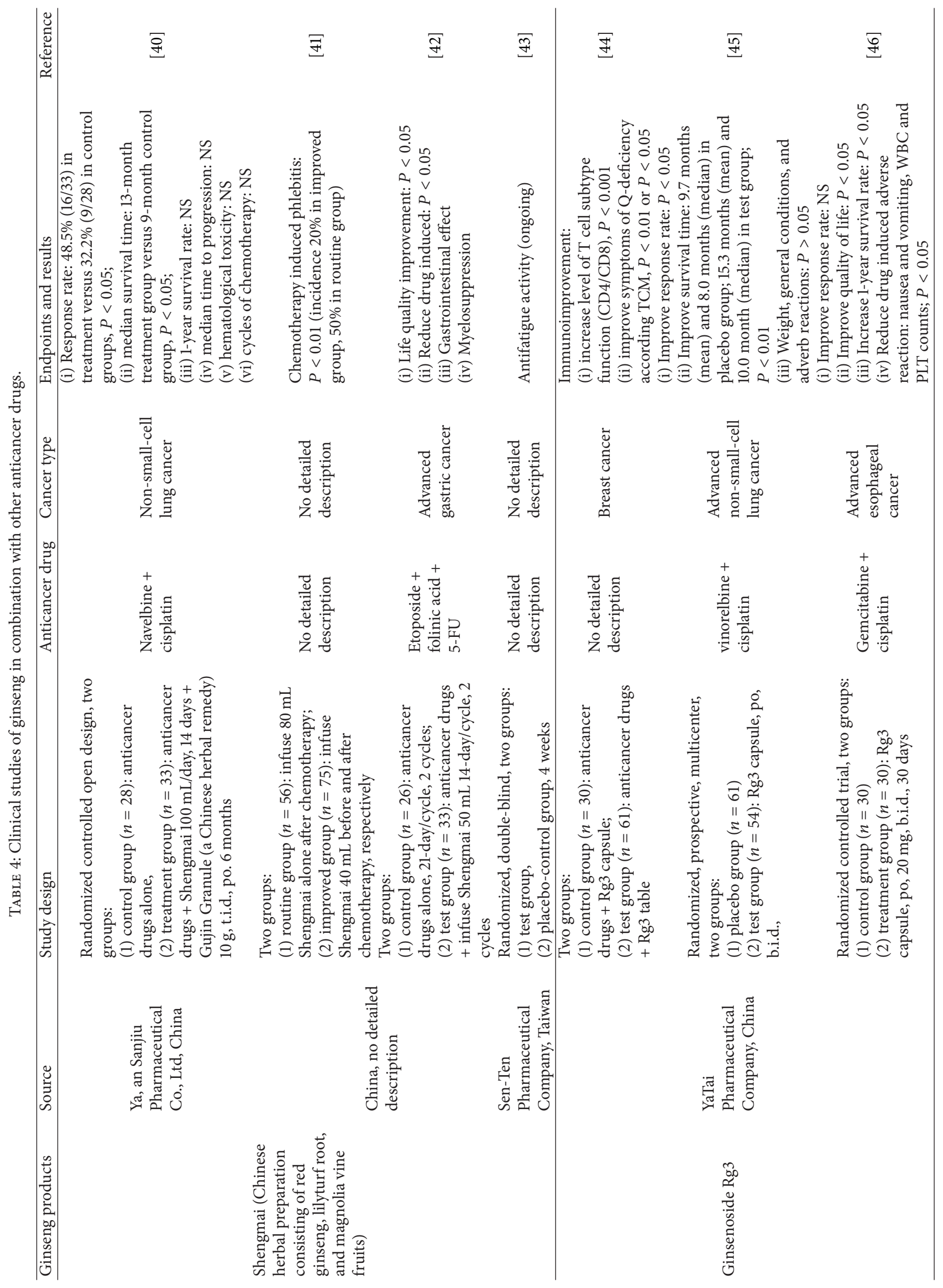


expression of iNOS and lipid peroxidation [39]. Furthermore, Shengmai has been found to also protect liver and renal function and increase white blood cell, platelet counts, and serum alanine aminotransferase [38]. Also, ginseng is known to modulate the immune system and thus may improve chemotherapy by an "indirect effect." Shim et al. have shown Korean ginseng can increase the expression level of the cytokines, such as TNF- $\alpha$, IL- $1 \beta$, IL- 6 , SCF and, GMCSF [32]. Polysaccharides may also improve drug-induced immunosuppression. They can significantly increase relative spleen weight (spleen weight/100 g of bodyweight, e.g., frm $2.89 \%$ cyclophosphamide alone to $3.42 \%$ combined use), stimulate lymphocyte proliferation, NK cell cytotoxicity, and macrophage activity, increase serum TNF- $\alpha$, IL-12, IFN- $\gamma$, and CRP (serum C-reactive protein) levels, and so forth $[27,31,33]$. However whether the degree of elevation of these cytokines by ginseng can improve chemotherapy outcome needs further investigated.

4.3. Clinical Studies. Despite popular use of ginseng, only a limited number of clinical studies have been reported on ginseng-chemotherapeutic agent combination (see Table 4). On the basis of the traditional Chinese medicine (TCM) consideration, the Chinese ginseng preparation Shengmai is selected as a tonic in combination with chemotherapy, for example, supplementing qi (means vital energy) and nourishing yin (means passive force) [40]. Chen et al. have evaluated the efficacy and side effects of Shengmai combined with chemotherapeutic agents in treating advanced non-small-cell lung cancer (NSCLC) [40]. This study was conducted on 63 patients with stages III B and IV NSCLC receiving navelbine (trade name of Vinorelbine, a plant alkaloid) and cisplatin chemotherapy. The patients were assigned to two groups: 33 patients in the treatment group receiving Shengmai by intravenous drip and Gujin Granule (a Chinese herbal remedy in water soluble granule) orally and 30 patients in the control group. Among the 61 patients (33 from the treatment group and 28 from the control group) who completed the observation, the response rate was $48.5 \%$ $(16 / 33)$ in the treatment and $32.2 \%(9 / 28)$ in the control groups, with a median survival time of 13 months and 9 months, respectively. This study indicated that the combined use of ginseng and anticancer drug might enhance the shortterm therapeutic efficacy of NSCLC. This study however was not blinded. A randomized, double-blind, placebo-controlled trial evaluating the therapeutic efficacy of Shenmai (same as Shengmai here) in cancer patients undergoing chemotherapy or radiotherapy is ongoing [43].

In another randomized controlled trial, Huang et al. evaluated the efficacy of Shenyi (95\% ginsenoside $\mathrm{Rg} 3$ ) in combination with gemcitabine plus cisplatin in 60 patients with advanced esophageal cancer. Compared to patients in the control group with chemotherapy alone, the results showed no significant difference in total response rate between the two groups during the treatment phase. After treatment, the vascular endothelial growth factor in the treatment group was found to be lower than that in the control group $(P<0.05)$, suggesting an effect of inhibiting angiogenesis.
In addition, one-year survival rate in the treatment group was higher compared with the control group $(P<0.05)$. Shenyi also improved the patients' quality of life according to the Karnofsky performance status scale [46]. $\operatorname{Rg} 3$ had been shown to have some anticancer activities like antiproliferative, apoptotic, antiangiogenic, antimetastatic, and antiinvasive effects as well as cell cycle regulation $[8,11,12]$.

\section{Summary and Conclusion}

Ginseng has been used primarily as a tonic to benefit cancer patients, especially in Asia. Based on our review of published in vitro, in vivo, and human studies, ginseng has excellent potential as a chemotherapy adjuvant, because of its low toxicity and many desirable properties such as antiangiogenesis, antiproliferation, anti-inflammation, antioxidation, apoptosis, and immune modulation effects [63]. Although there are substantial evidence from in vitro and animal studies showing the benefit of ginseng and its active constituents in enhancing antitumor activity when used in combination with other anticancer drugs, there is insufficient clinical evidence of such benefit at present.

Ginseng has already been accepted as a natural product for health promotion. For this reason, continued use of ginseng together with encouraging results from the in vitro and in vivo animal studies (see Tables 2 and 3) may provide important clues to demonstrate future clinic benefit of ginseng. Further studies of ginseng products should include quality control such as the use of activity markers and active components, as well as determination of their pharmacokinetics and pharmacodynamics. It is hoped that government support as well as development of new process patents for ginseng will provide sufficient incentive and funding to conduct well designed clinical trials leading to regulatory approval of a ginseng product for chemotherapy enhancement in the future.

\section{Conflict of Interests}

The authors declare that there is no conflict of interests.

\section{References}

[1] C. T. Che, Z. J. Wang, M. S. S. Chow, and C. W. K. Lam, "Herbherb combination for therapeutic enhancement and advancement: theory, practice and future perspectives," Molecules, vol. 18, pp. 5125-5141, 2013.

[2] Z. J. Wang, C. Xie, Y. Huang, C. W. K. Lam, and M. S. S. Chow, "Overcoming chemotherapy resistance with herbal medicines: past, present and future perspectives," Phytochemistry Reviews, vol. 13, no. 1, pp. 323-337, 2014.

[3] F. Qi, A. Li, Y. Inagaki et al., "Chinese herbal medicines as adjuvant treatment during chemo- or radio-therapy for cancer," BioScience Trends, vol. 4, no. 6, pp. 297-307, 2010.

[4] B. K. Vogler, M. H. Pittler, and E. Ernst, "The efficacy of ginseng. A systematic review of randomised clinical trials," European Journal of Clinical Pharmacology, vol. 55, no. 8, pp. 567-575, 1999. 
[5] J. Lü, Q. Yao, and C. Chen, "Ginseng compounds: an update on their molecular mechanisms and medical applications," Current Vascular Pharmacology, vol. 7, no. 3, pp. 293-302, 2009.

[6] A. T. Smolinski and J. J. Pestka, "Modulation of lipopolysaccharide-induced proinflammatory cytokine production in vitro and in vivo by the herbal constituents apigenin (chamomile), ginsenoside $\mathrm{Rbl}$ (ginseng) and parthenolide (feverfew)," Food and Chemical Toxicology, vol. 41, no. 10, pp. 1381-1390, 2003.

[7] M. Blumenthal, "Herb sales down 15 percent in mainstream market," HerbalGram, no. 51, article 69, 2001.

[8] S. A. Nag, J. J. Qin, W. Wang, M. H. Wang, H. Wang, and R. Zhang, "Ginsenosides as anticancer agents: in vitro and in vivo activities, structure-activity relationships, and molecular mechanisms of action," Frontiers in Pharmacology, vol. 3, no. 25, pp. 1-18, 2012.

[9] D. W. Kaufman, J. P. Kelly, L. Rosenberg, T. E. Anderson, and A. A. Mitchell, "Recent patterns of medication use in the ambulatory adult population of the United States: the Slone survey," The Journal of the American Medical Association, vol. 287, no. 3, pp. 337-344, 2002.

[10] T. Yun and S. Choi, "Preventive effect of ginseng intake against various human cancers: a case-control study on 1987 pairs," Cancer Epidemiology Biomarkers and Prevention, vol. 4, no. 4, pp. 401-408, 1995.

[11] T. Liu, Y. Huang, D. Cui et al., "Inhibitory effect of ginsenoside $\mathrm{Rg} 3$ combined with gemcitabine on angiogenesis and growth of lung cancer in mice," BMC Cancer, vol. 9, article 250, 2009.

[12] H. Q. Hua, X. K. Shen, S. K. Qin, and H. Y. Chen, "Antiinvasion and anti-metastasis effects of ginsenoside $\mathrm{Rg} 3$ on the hepatocellular carcinoma cell line," Zhongguo Ai Zheng Za Zhi, vol. 15, no. 4, pp. 326-330, 2005.

[13] K. W. Leung, L. W. T. Cheung, Y. L. Pon et al., "Ginsenoside $\mathrm{Rbl}$ inhibits tube-like structure formation of endothelial cells by regulating pigment epithelium-derived factor through the oestrogen $\beta$ receptor," British Journal of Pharmacology, vol. 152, no. 2, pp. 207-215, 2007.

[14] A. Papapetropoulos, "A ginseng-derived oestrogen receptor $\beta$ $(\mathrm{ER} \beta)$ agonist, Rb1 ginsenoside, attenuates capillary morphogenesis," British Journal of Pharmacology, vol. 152, no. 2, pp. 172174, 2007.

[15] A. B. Fishbein, C. Wang, X. Li et al., "Asian ginseng enhances the anti-proliferative effect of 5-fluorouracil on human colorectal cancer: comparison between white and red ginseng," Archives of Pharmacal Research, vol. 32, no. 4, pp. 505-513, 2009.

[16] C. X. Gu, J. P. Qiao, M. L. Zhu et al., "Preliminary evaluation of the interactions of Panax ginseng and Salvia miltiorrhiza Bunge with 5-fluorouracil on pharmacokinetics in rats and pharmacodynamics in human cells," The American Journal of Chinese Medicine, vol. 41, no. 2, pp. 443-458, 2013.

[17] Y. S. Chang, E. Seo, C. Gyllenhaal, and K. I. Block, "Panax ginseng: a role in cancer therapy?" Integrative Cancer Therapies, vol. 2, no. 1, pp. 13-33, 2003.

[18] G. Du, C. Wang, Z. Zhang et al., "Caspase-mediated proapoptotic interaction of panaxadiol and irinotecan in human colorectal cancer cells," Journal of Pharmacy and Pharmacology, vol. 64, no. 5, pp. 727-734, 2012.

[19] X. Li, C. Wang, S. R. Mehendale, S. Sun, Q. Wang, and C. Yuan, "Panaxadiol, a purified ginseng component, enhances the anti-cancer effects of 5-fluorouracil in human colorectal cancer cells," Cancer Chemotherapy and Pharmacology, vol. 64, no. 6, pp. 1097-1104, 2009.
[20] H. Matsunaga, M. Katano, T. Saita, H. Yamamoto, and M. Mori, "Potentiation of cytotoxicity of mitomycin C by a polyacetylenic alcohol, panaxytriol," Cancer Chemotherapy and Pharmacology, vol. 33, no. 4, pp. 291-297, 1994.

[21] S. M. Kim, S. Y. Lee, D. Y. Yuk et al., "Inhibition of NF- $\kappa$ B by ginsenoside Rg3 enhances the susceptibility of colon cancer cells to docetaxel," Archives of Pharmacal Research, vol. 32, no. 5, pp. 755-765, 2009.

[22] S. M. Kim, S. Y. Lee, J. S. Cho et al., "Combination of ginsenoside Rg3 with docetaxel enhances the susceptibility of prostate cancer cells via inhibition of NF- $\kappa \mathrm{B}$," European Journal of Pharmacology, vol. 631, no. 1-3, pp. 1-9, 2010.

[23] R. Gao, J. Jin, and Y. Niu, "Potentiated effects of total saponins of Panax ginseng on inhibition of leukemic cells by cytotoxic drugs," Zhongguo Zhong Xi Yi Jie He Za Zhi, vol. 19, no. 1, pp. 17-19, 1999.

[24] S. H. Baek, X. L. Piao, U. J. Lee, H. Y. Kim, and J. H. Park, "Reduction of cisplatin-induced nephrotoxicity by ginsenosides isolated from processed ginseng in cultured renal tubular cells," Biological and Pharmaceutical Bulletin, vol. 29, no. 10, pp. 20512055, 2006

[25] T. Yokozawa and E. Dong, "Role of ginsenoside-Rd in cisplatininduced renal injury: special reference to DNA fragmentation," Nephron, vol. 89, no. 4, pp. 433-438, 2001.

[26] C. Choi, G. Kang, and Y. Min, "Reversal of P-glycoproteinmediated multidrug resistance by protopanaxatriol ginsenosides from Korean red ginseng," Planta Medica, vol. 69, no. 3, pp. 235-240, 2003.

[27] M. J. Shin, Y. S. Kim, Y. S. Kwak, Y. B. Song, Y. S. Kim, and J. D. Park, "Enhancement of antitumor effects of paclitaxel (taxol) in combination with red ginseng acidic polysaccharide (RGAP)," Planta Medica, vol. 70, no. 11, pp. 1033-1038, 2004.

[28] M. Miao, Q. Liu, and Y. R. Liu, "Chemo-sensitivity enhancing effects of Shengai injection on various chemotherapeutic drugs," Chinese Traditional and Herbal Drugs, vol. 44, no. 7, pp. 875876, 2013.

[29] H. R. B. Raghavendran, S. Rekha, J. Shin et al., "Effects of Korean ginseng root extract on cisplatin-induced emesis in a rat-pica model," Food and Chemical Toxicology, vol. 49, no. 1, pp. 215221, 2011.

[30] J. Kim, I. Yoon, B. Lee et al., "Effects of Korean red ginseng extract on cisplatin-induced nausea and vomiting," Archives of Pharmacal Research, vol. 28, no. 6, pp. 680-684, 2005.

[31] X. F. Du, C. Z. Jiang, C. F. Wu, E. K. Won, and S. Y. Choung, "Synergistic immunostimulating activity of pidotimod and red ginseng acidic polysaccharide against cyclophosphamideinduced immunosuppression," Archives of Pharmacal Research, vol. 31, no. 9, pp. 1153-1159, 2008.

[32] J. Y. Shim, Y. Han, J. Y. Ahn, Y. S. Yun, and J. Song, "Chemoprotective and adjuvant effects of immunomodulator ginsan in cyclophosphamide-treated normal and tumor bearing mice," International Journal of Immunopathology and Pharmacology, vol. 20, no. 3, pp. 487-497, 2007.

[33] W. Ni, X. Zhang, B. Wang et al., "Antitumor activities and immunomodulatory effects of ginseng neutral polysaccharides in combination with 5-fluorouracil," Journal of Medicinal Food, vol. 13, no. 2, pp. 270-277, 2010.

[34] T. Xu, Y. Xin, M. Cui, X. Jiang, and L. Gu, "Inhibitory effect of ginsenoside Rg3 combined with cyclophosphamide on growth and angiogenesis ovarian cancer," Chinese Medical Journal, vol. 120, no. 7, pp. 584-588, 2007. 
[35] E. Ernst, "Panax ginseng: an overview of the clinical evidence," Journal of Ginseng Research, vol. 34, no. 4, pp. 259-263, 2010.

[36] Q. Zhang, X. Kang, and W. Zhao, "Antiangiogenic effect of low-dose cyclophosphamide combined with ginsenoside Rg3 on Lewis lung carcinoma," Biochemical and Biophysical Research Communications, vol. 342, no. 3, pp. 824-828, 2006.

[37] B. Kenarova, H. Neychev, C. Hadjiivanova, and V. D. Petkov, "Immunomodulating activity of ginsenoside Rgl from Panax ginseng," Japanese Journal of Pharmacology, vol. 54, no. 4, pp. 447-454, 1990.

[38] Z. Chen, P. Wang, W. Huang, and L. Liu, "Experimental study on effects of shengmai injection: enhancing 5-FU anti-tumor efficacy and reducing its toxicity," Journal of Chinese Integrative Medicine, vol. 3, no. 6, pp. 476-479, 2005.

[39] M. Ge, Y. Y. Fang, G. P. Liu, and S. D. Guan, "Effect of Shengmai injection on diaphragmatic contractility in doxorubicin-treated rats," Chinese Journal of Integrative Medicine, vol. 20, no. 1, pp. 43-48, 2014.

[40] Y. Chen, Z. Li, F. Gao, Y. Zhang, H. Sun, and P. Li, "Effects of combined Chinese drugs and chemotherapy in treating advanced non-small cell lung cancer," Chinese Journal of Integrative Medicine, vol. 15, no. 6, pp. 415-419, 2009.

[41] A. Y. Yan, S. J. Guo, Y. J. Yi et al., "Effect of Shengmai injection "Sandwich" infusion to prevent patients with chemotherapy induced phlebitis," Chinese Nursing Research, vol. 18, no. 20, pp. 1859-1860, 2004.

[42] Z. Zhao, Z. J. Liao, and X. H. Zhao, "The clinic research of the effect of SHENG MAI injection combined with the chemotherapy in the elderly patients with advanced gastric cancer," Journal of Mordern Oncology, vol. 13, no. 3, pp. 387-388, 2005.

[43] L. C. Lo, C. Y. Chen, S. T. Chen, H. C. Chen, T. C. Lee, and C. S. Chang, "Therapeutic efficacy of traditional Chinese medicine, Shen-Mai San, in cancer patients undergoing chemotherapy or radiotherapy: study protocol for randomized, double-blind, placebo-controlled trial," Trials, vol. 13, article 232, 2012.

[44] J. W. Liu, L. X. Sun, Y. Zhao et al., "Clinical phase II study on immunoimprovement of patients with breast cancer treated by Shengyi capsule," Chinese Journal of Clinical Oncology, vol. 27, no. 7, pp. 534-544, 2000.

[45] Y. Sun, H. Zhu, Y. Zhu et al., "A randomized, prospective, multicentre clinical trial of NP regimen (vinorelbine+cisplatin) plus Gensing Rg3 in the treatment of advanced non-small cell lung cancer patients," Chinese Journal of Lung Cancer, vol. 9, no. 3, pp. 254-258, 2006.

[46] J. Huang, Y. Sun, Q. Fan, and Y. Zhang, "Efficacy of Shenyi Capsule combined with gemcitabine plus cisplatin in treatment of advanced esophageal cancer: a randomized controlled trial," Journal of Chinese Integrative Medicine, vol. 7, no. 11, pp. 10471051, 2009.

[47] J. T. Coon and E. Ernst, "Panax ginseng: a systematic review of adverse effects and drug interactions," Drug Safety, vol. 25, no. 5, pp. 323-344, 2002.

[48] L. Qi, C. Wang, G. Du, Z. Zhang, T. Calway, and C. Yuan, "Metabolism of ginseng and its interactions with drugs," Current Drug Metabolism, vol. 12, no. 9, pp. 818-822, 2011.

[49] D. G. Popovich, C. R. Yeo, and W. Zhang, "Ginsenosides derived from Asian (Panax ginseng), American ginseng (Panax quinquefolius) and potential cytoactivity," International Journal of Biomedical and Pharmaceutical Sciences, vol. 6, no. 1, pp. 5662, 2012.
[50] T. K. Yun, "Brief introduction of Panax ginseng C.A. Meyer," Journal of Korean Medical Science, vol. 16, supplement, pp. S3S5, 2001.

[51] T. Yun, "Panax ginseng-a non-organ-specific cancer preventive?” The Lancet Oncology, vol. 2, no. 1, pp. 49-55, 2001.

[52] H. R. Shin, J. Y. Kim, T. K. Yun, G. Morgan, and H. Vainio, "The cancer-preventive potential of Panax ginseng: a review of human and experimental evidence," Cancer Causes and Control, vol. 11, no. 6, pp. 565-576, 2000.

[53] S. Kang and H. Min, "Ginseng, the "immunity boost": the effects of Panax ginseng on immune system," Journal of Ginseng Research, vol. 36, no. 4, pp. 354-368, 2012.

[54] S. Lim, C. Cho, U. Choi, and Y. Kim, "Antioxidant activity and ginsenoside pattern of fermented white ginseng," Journal of Ginseng Research, vol. 34, no. 3, pp. 168-174, 2010.

[55] M. R. Harkey, G. L. Henderson, M. E. Gershwin, J. S. Stern, and R. M. Hackman, "Variability in commercial ginseng products: an analysis of 25 preparations," American Journal of Clinical Nutrition, vol. 73, no. 6, pp. 1101-1106, 2001.

[56] M. Mizuno, J. Yamada, H. Terai, N. Kozukue, Y. S. Lee, and H. Tsuchida, "Differences in immunomodulating effects between wild and cultured Panax ginseng," Biochemical and Biophysical Research Communications, vol. 200, no. 3, pp. 1672-1678, 1994.

[57] X. Zhang, L. Yu, H. Bi et al., "Total fractionation and characterization of the water-soluble polysaccharides isolated from Panax ginseng C. A. Meyer,' Carbohydrate Polymers, vol. 77, no. 3, pp. 544-552, 2009.

[58] X. G. Gao, Y. Zhi, L. Sun et al., "The inhibitory effects of a rhamnogalacturonan I, (RG-I) domain from ginseng pectin on galectin-3 and its structure-activity relationship," The Journal of Biological Chemistry, vol. 288, no. 47, pp. 33953-33965, 2013.

[59] Y. Chu, H. C. Zhang, S. M. Li et al., "Determination of ginsenoside Rc in rat plasma by LC-MS/MS and its application to a pharmacokinetic study," Journal of Chromatography B: analytical Technologies in the Biomedical and Life Sciences, vol. 919-920, pp. 75-78, 2013.

[60] C. Lv, Q. Li, Y. Zhang et al., "A UFLC-MS/MS method with a switching ionization mode for simultaneous quantitation of polygalaxanthone III, four ginsenosides and tumulosic acid in rat plasma: application to a comparative pharmacokinetic study in normal and Alzheimer's disease rats," Journal of Mass Spectrometry, vol. 48, no. 8, pp. 904-913, 2013.

[61] A. Sparreboom, M. C. Cox, M. R. Acharya, and W. D. Figg, "Herbal remedies in the United States: potential adverse interactions with anticancer agents," Journal of Clinical Oncology, vol. 22, no. 12, pp. 2489-2503, 2004.

[62] B. X. Wang, J. C. Cui, and A. J. Liu, "The action of ginsenosides extracted from the stems and leaves of Panax ginseng in promoting animal growth," Acta Pharmaceutica Sinica, vol. 17, no. 12 , pp. 899-904, 1982.

[63] S. Helms, "Cancer prevention and therapeutics: Panax ginseng", Alternative Medicine Review, vol. 9, no. 3, pp. 259-274, 2004. 


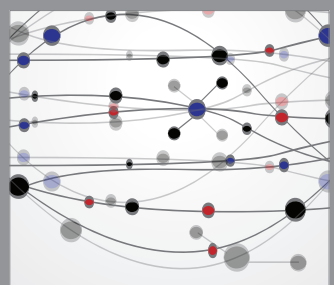

The Scientific World Journal
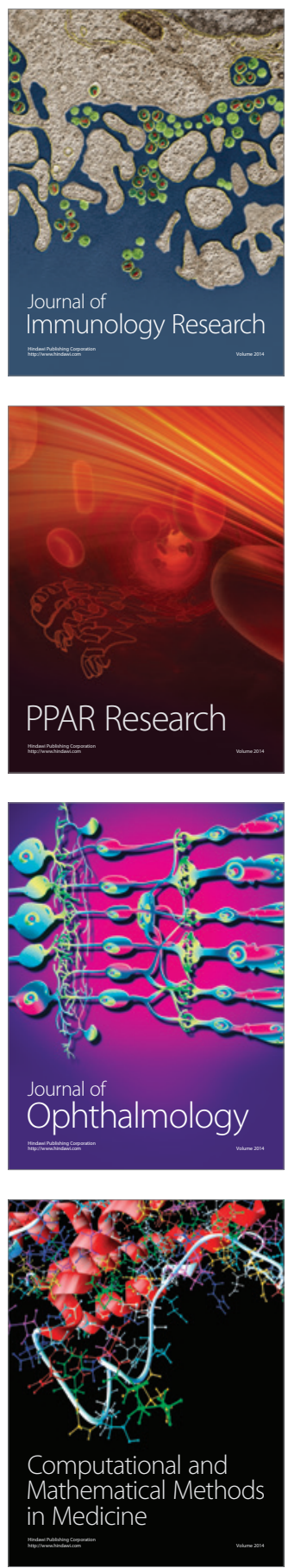

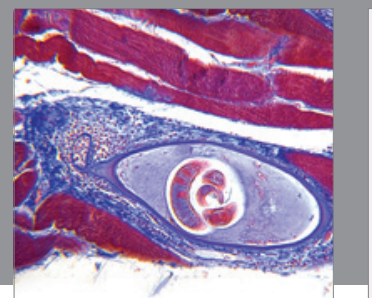

Gastroenterology

Research and Practice
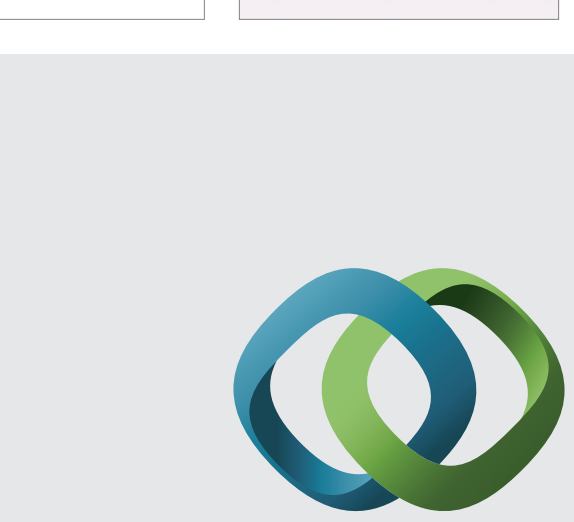

\section{Hindawi}

Submit your manuscripts at

http://www.hindawi.com
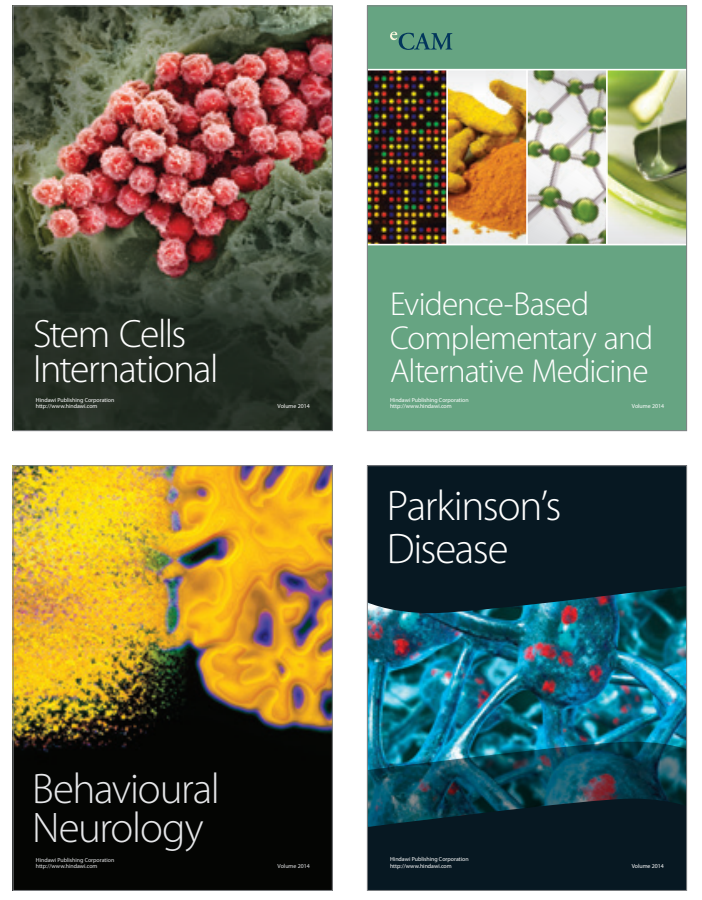
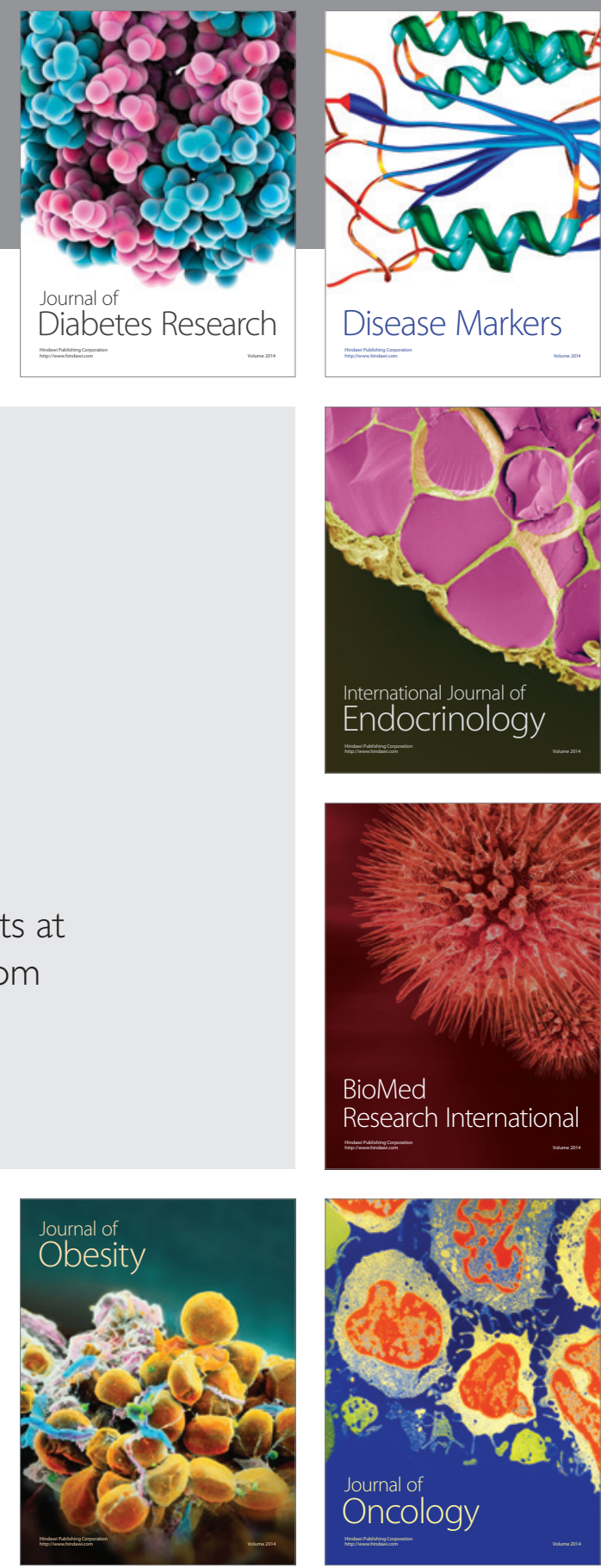

Disease Markers
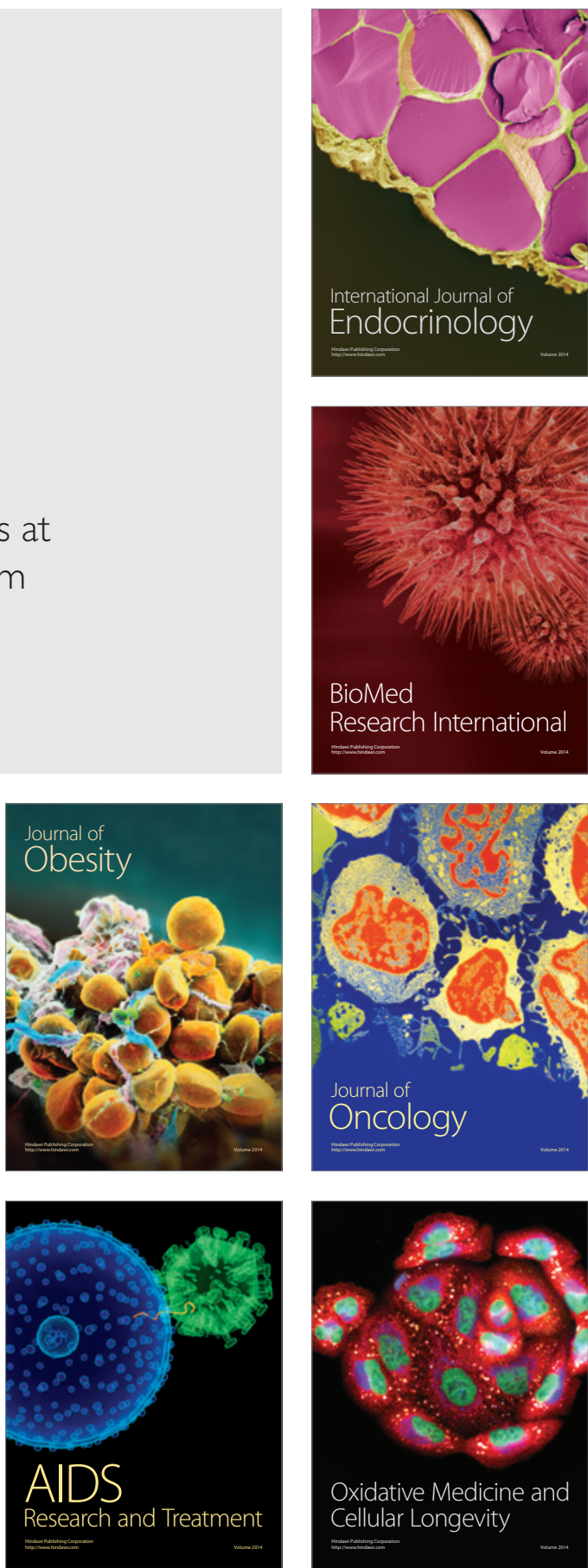